Recherche et formation

\title{
Les situations de travail
}

Références pour les référentiels

Work situations : References for Systems of Reference

Las situaciones de trabajo : referencias para los referenciales

Die Arbeitssituationen : Referenzen für die Bezugssysteme

Patrick Mayen, Jean-François Métral et Claire Tourmen

\section{OpenEdition}

Journals

Édition électronique

URL : http://journals.openedition.org/rechercheformation/191

DOI : 10.4000/rechercheformation.191

ISSN : 1968-3936

Éditeur

ENS Éditions

\section{Édition imprimée}

Date de publication : 1 juillet 2010

Pagination : $31-46$

ISSN : 0988-1824

Référence électronique

Patrick Mayen, Jean-François Métral et Claire Tourmen, « Les situations de travail », Recherche et formation [En ligne], 64 | 2010, mis en ligne le 01 juillet 2012, consulté le 30 avril 2019. URL : http:// journals.openedition.org/rechercheformation/191; DOI : 10.4000/rechercheformation.191 


\title{
Les situations de travail
}

\section{Références pour les référentiels}

\author{
$>$ Patrick MAYEN \\ $>$ Jean-François MÉTRAL \\ $>$ Claire TOURMEN \\ AgroSup Dijon, Institut national supérieur des sciences agronomiques \\ de l'alimentation et de l'environnement
}

\begin{abstract}
RÉSUMÉ - Cet article propose une approche des référentiels à partir de la didactique professionnelle et de la place fondatrice que celle-ci accorde aux situations de travail. En formation professionnelle, ce qui est premier, ce sont les situations parce que ce sont celles-ci dont les professionnels et futurs professionnels ont ou auront à " se débrouiller ". La nécessité d'identifier puis de caractériser les situations, notamment dans les exigences qu'elles imposent à l'activité, constitue une étape antérieure à l'analyse et à la description de l'activité, et plus antérieure encore à l'analyse et à la description des ressources utiles pour penser et agir en situation. Dans cette approche, les situations de travail constituent la part absente et méconnue des référentiels.
\end{abstract}

MOTS-CLÉS • compétence, situation, travail, activité, didactique

Une des voies possibles pour réduire les confusions engendrées par la primauté accordée à la notion de compétence consiste à la mettre au second plan des préoccupations, en commençant par ne pas en faire une substance. Le premier pas dans ce sens consiste à ne pas définir la compétence mais à partir de la définition de ce qu'est un professionnel compétent : " qu'est-ce qu'un professionnel compétent? Définition 1: A est plus compétent que $B$ s'il sait faire quelque chose que $B$ ne sait pas faire [...]. Définition 2 : A est plus compétent que B s'il s'y prend d'une meilleure manière. Le comparatif " meilleur " suppose des critères complémentaires : rapidité, fiabilité, économie, élégance, etc. Définition 3 : A est plus compétent s'il dispose d'un répertoire de ressources alternatives qui lui permet d'utiliser tantôt une procédure, tantôt une autre, et de s'adapter ainsi plus aisément aux différents cas de figure qui peuvent se présenter. Définition $4:$ A est plus compétent s'il sait se débrouiller devant une situation nouvelle d'une catégorie jamais rencontrée auparavant. "(Vergnaud, 2001, p. 2). 
Nous retiendrons de ces définitions que la compétence n'est pas une substance mais la relation dynamique d'une personne avec des situations ou des classes de situations. Si toute situation de travail est unique, elle comporte un certain nombre de traits génériques qui l'assimilent aux autres situations de la même classe (Pastré, Mayen \& Vergnaud, 2006) et rend possible leur description.

Le deuxième pas consiste à penser la formation professionnelle d'une manière différente de la formation initiale. En formation professionnelle, les courants de la didactique professionnelle et de l'analyse de "l'activité orientée formation " font des situations de travail la finalité et l'origine de la formation professionnelle. Ce sont, en effet, les situations qui sont premières parce que ce sont elles dont les professionnels ou futurs professionnels ont, ou auront, à se débrouiller dans le cours de leur vie professionnelle, aussi bien pour les identifier et les connaître que pour comprendre comment elles interagissent avec et sur leur propre action, et comment ils peuvent agir sur elles (Samurçay \& Rogalski, 1993 ; Mayen, 2007).

Dans la démarche de conception d'un référentiel comme dans la démarche de conception d'un dispositif de formation, l'analyse du travail est donc première. II s'agit alors d'identifier et de caractériser d'une part, les situations, et d'autre part, l'activité des professionnels, plus ou moins expérimentés et compétents (au sens des définitions de Vergnaud, ibid.), engagés dans ces situations.

Dans cet article, nous allons présenter une approche de didactique professionnelle pour la conception de référentiels de situations. Nous nous appuierons sur les résultats de trois recherches, qui ont mobilisé le cadre théorique de la didactique professionnelle, en vue de la construction de tels référentiels. Elles concernent des champs professionnels distincts (l'aquaculture, l'agronomie/agroalimentaire, l'évaluation des politiques publiques) et des niveaux de formation différents (BTS, formation d'ingénieurs et formations universitaires de Master 2).

\section{La didactique professionnelle et la compétence des professionnels}

\subsection{Référentialiser la compétence?}

Lorsque l'on se préoccupe de concevoir des référentiels de compétences, une idée implicite, fréquente et active est qu'il faudrait chercher dans les individus des compétences valables pour toute une population de professionnels. On cherche alors à décrire des ressources, "ressources " individuelles que les professionnels devraient " détenir " pour réaliser les tâches attendues. Cela aboutit, le plus souvent, à découper l'activité des professionnels en autant de tâches listées qui deviennent des " savoir-faire " (" capacité à "), puis à identifier des " savoirs " et " savoir-être " qui seraient la propriété des professionnels, et donc évaluables et transférables. Les référentiels de compétences présentent alors des listes de capacités, connaissances, savoir-faire, savoir-être, habiletés, aptitudes, qualités, etc. et compétences, plus ou 
moins généraux, décontextualisés et non hiérarchisés. Stroobants (1994) parlait d'un " ensemble hétéroclite d'aptitudes, de savoirs, de capacités d'action et de comportements " et Zarifian (2001, p. 18) soulignait les limites des " descriptifs et classifications d'emplois, associés à des requis de capacités, auxquels on ajoute une couche de savoir-être " et que l'on " baptise : un référentiel de compétences ". Dans ce cas, rien - ou si peu - n'est dit sur la réalité du travail, ses contraintes, ses objets, autrement dit, les situations que les professionnels auront à rencontrer.

Il faut d'ailleurs souligner que ce n'est plus la voie choisie par les grands certificateurs publics, notamment l'Éducation nationale, dont les référentiels, et en particulier les nouveaux référentiels de BTS, évitent soigneusement cet écueil. Depuis longtemps, les "référentiels métiers " et les "référentiels d'activité " des diplômes de l'enseignement professionnel, partent d'abord des contextes socioéconomiques, des emplois et des fonctions, puis des activités, de plus en plus " situées ". C'est aussi le cas de certaines approches développées en Amérique du Nord et au niveau international (Jonnaert et al. 2006).

La didactique professionnelle propose une approche qui accorde la première place aux situations et à l'activité en situation. Dans cette perspective, la question des compétences n'est jamais première. La description, la définition (et le choix des mots pour le faire) des " ressources " pour l'action découle de l'analyse des situations et de l'activité en situation.

\subsection{La didactique professionnelle : une approche par les situations}

La didactique professionnelle part du travail dans et sur des situations professionnelles. Être compétent pour une situation ou une classe de situations consiste alors à pouvoir s'en débrouiller, faire face, se tirer d'affaire, parfois même maîtriser la situation. Autrement dit, c'est pouvoir agir avec elle, sur elle, ne pas être seulement passif et réactif, ne pas trop en subir les aléas, les événements, les conséquences. Un professionnel compétent est alors une personne capable de " maîtriser " un ensemble de situations professionnelles, et, par extension, de classes de situations, plus ou moins complexes, constitutives de son emploi ou métier (Leplat, 1991 ; Vergnaud, 1990, 1996 ; Pastré, 2004 ; Ferron et al. 2006).

Cette définition rappelle que la compétence est située, c'est-à-dire toujours relative à une situation ou à une classe de situations. Diversité et variabilité distinguent les multiples formes d'une classe de situations, "toujours identiques et toujours différentes " (Mayen, 2004). D'un poste à un autre, les conditions du travail pour deux situations de la même classe peuvent être différentes. Dans une situation singulière, même procéduralisée et contrainte fortement par un système technique, chaque occurrence de la tâche peut varier par rapport à un état " normal " (variations de l'état de fatigue et d'attention de celui qui travaille, du produit à fabriquer, des clients...). Le professionnel compétent est alors amené à composer avec ces variations. 
Dès lors, comme le rappellent Ferron et al. (2006, p. 15-16), " ce qu'une personne peut faire dans une situation donnée est fonction des ressources et contraintes de la situation et du rapport que la personne entretient avec celle-ci. Les caractéristiques de situation peuvent, en effet, limiter ou inhiber les capacités d'action, les mobiles propres à une personne (ce qui la motive) ou bien, à l'inverse, ouvrir des voies d'investissement [...], fournir des ressources matérielles, instrumentales, des aides d'autrui par lesquelles les compétences sont développées ". Cette remarque insiste sur le caractère distribué de la compétence dans la situation : l'individu dispose-t-il des moyens suffisants pour agir efficacement? Les règles sont-elles suffisamment claires pour agir en conformité ? N'est-il pas face à des injonctions implicites, voire contradictoires ? Est-il reconnu à sa juste valeur pour que sa motivation à faire du " beau travail " soit maintenue ? Cela ne braque pas le faisceau uniquement sur "les ressources" des individus et ne leur renvoie pas la seule responsabilité de leur compétence. Ils peuvent être plus ou moins compétents pour, avec, et parfois malgré les conditions de leur travail.

Cependant, contrairement à ce qu'une conception trop radicale de l'action située pourrait nous laisser penser (Rogalski, 2004), réaffirmer la dimension située des compétences ne revient pas à nier qu'il y a du générique dans les situations. Toute situation comporte un certain nombre de traits génériques qui l'assimilent aux autres situations de la même classe, permettent la reconnaissance de la classe de situations et donc, l'orientation et l'organisation de l'action dans la situation. C'est ce dont rend compte la théorie des schèmes (Vergnaud 1990, 1992, 1996). Le schème, que l'on peut définir comme une organisation invariante de l'activité pour une classe de situations donnée, permet de rendre compte de l'invariance et des régularités qui permettent l'action en situation et d'y articuler des possibilités d'adaptation et de flexibilité face à la variabilité et la diversité entre les situations singulières qui constituent une même classe (Pastré, 2002 ; Mayen, 2004 ; Ferron, Mayen et al., 2006). Ce qui revient à dire qu'être compétent, c'est être capable " de faire face à plus de variations plus importantes, en particulier en étant capable d'identifier l'état des variables les plus essentielles d'une situation (état de l'objet du travail, état des conditions de réalisation de l'action) et en disposant d'un ensemble de connaissances sur ces états qui permettent de les combiner et de construire une configuration significative à partir de laquelle il sera possible de décider d'une action à entreprendre. " (Ferron et al., 2006, p. 20).

L'un des objectifs du travail d'élaboration d'un référentiel de situations est alors de mettre au jour les éléments invariants de la classe de situations et les variables les plus essentielles, autrement dit de sa structure conceptuelle (Pastré, 1999), c'està-dire de l'ensemble des concepts organisant l'action et servant à la guider chez les sujets ayant une activité efficace. Ces invariants portent sur les traits caractéristiques des différents composants d'une situation : but(s), objet(s) à transformer, conditions de réalisation. 


\section{Les référentiels de situations}

Nous allons maintenant présenter les conséquences que nous avons tirées de cette approche en matière d'ingénierie des référentiels.

\subsection{Construire des référentiels de situations}

Dans cette perspective, ce qui est premier ce sont les situations qu'un professionnel sera susceptible de rencontrer. L'objectif devient alors de mettre en évidence, au-delà des traits de surface souvent trompeurs par lesquels on croit connaître un travail, les principales caractéristiques agissantes de ces situations. Les " ressources" mobilisées par les professionnels, parmi lesquelles les savoirs scientifiques disciplinaires, prennent ainsi un sens différent au sein de leur activité : " celui d'instrumenter la compréhension des situations à partir de l'expérience des situations, celui d'instrumenter l'action en situation à partir des tâches et problèmes à résoudre " (Ferron, Mayen et al., 2006, p. 12).

Cette entrée a des conséquences pour le recueil des données (traces de l'activité en situation des professionnels), pour leur analyse (identifier les variables agissantes) et sur la structure du référentiel construit qui devient un référentiel de situations.

Chaque emploi serait ainsi constitué d'un ensemble de classes de situations prototypiques ou caractéristiques, d'autres pouvant être annexes ou connexes. II s'agit alors " d'identifier " ces classes de situations, que nous appelons " situations génériques " d'un champ professionnel, puis de les caractériser. Une partie des éléments nécessaires peut, d'ailleurs, être trouvée dans les référentiels métiers existants. Ceux-ci donnent des indications sur les secteurs d'activité dans lesquels le titulaire du diplôme peut exercer, les types d'emplois et de fonctions accessibles, les tâches relatives à ces emplois et, de là, parfois, les situations à maîtriser dans ces emplois. Cependant, ces référentiels ne disent rien de ce qui organise l'activité, en particulier des variables les plus essentielles à prendre en compte dans l'activité efficace. Pour accéder à ces éléments, la porte d'entrée se trouve dans l'activité du sujet en situation : il faut recueillir et analyser des traces de cette activité pour plusieurs professionnels agissant dans une même classe de situation.

\subsection{Trois exemples de construction de référentiels de situations}

Nous avons tenté d'opérationnaliser cette approche dans trois référentiels de situations construits suite à trois recherches distinctes, menées au sein de l'équipe " Développement professionnel et formation " d'AgroSup Dijon. Une recherche portait sur des professionnels de l'aquaculture (Ferron, Mayen et al., 2006), l'autre sur des ingénieurs agronomes (Métral \& Mayen, 2008) et la dernière s’intéressait à des professionnels de l'évaluation de programmes et politiques publiques (Tourmen, 2007, 2008). 


\subsubsection{Recueillir des traces de l'activité professionnelle}

Ces trois recherches mobilisant le cadre théorique de la didactique professionnelle étaient basées sur un recueil et une analyse de traces de l'activité des professionnels concernés. Les traces, de différentes natures, correspondaient toujours à des situations singulières : situations relatives à un professionnel, dans une entreprise donnée, à un moment donné, etc. (voir le détail méthodologique dans l'encadré 1).

1. Les professionnels de l'aquaculture (Ferron, Mayen et al., 2006)

Analyse documentaire sur le contexte de l'aquaculture, dont quatre entretiens avec deux experts et treize entretiens en situation avec des aquaculteurs travaillant dans des contextes différents. Identification des situations génériques du métier avec un groupe d'experts.

Analyse approfondie de deux à quatre entretiens pour caractériser l'organisation de l'activité des professionnels rencontrés dans chaque situation générique.

2. Une recherche sur les ingénieurs agronomes (Métral \& Mayen, 2008)

Analyse de dix dossiers de Validation des acquis de l'expérience (VAE) de candidats à des diplômes d'ingénieur agronome et de cinq jurys de Validation des acquis de l'expérience. Identification, avec un groupe de travail issu de huit établissements de l'enseignement supérieur agronomique, des situations génériques de l'ingénieur, confirmées par une étude bibliographique.

Analyse des variables apparaissant essentielles aux candidats - et aux jurys - dans la conduite de l'activité d'ingénieur, et analyse des capacités attendues et testées par les jurys.

3. Une thèse sur les professionnels de l'évaluation de politiques publiques (Tourmen, 2007, 2008) Analyse des situations rencontrées par les évaluateurs : quatorze entretiens exploratoires avec des professionnels variés, analyse de la prescription et des théories du domaine d'activité. Analyse de l'activité d'un échantillon de professionnels de l'évaluation : neuf professionnels rencontrés (cinq expérimentés et quatre débutants) à l'aide de trois méthodes d'accès à leur activité : trois entretiens d'explicitation, observation de deux séquences de travail réel et quatre simulations avec entretiens cliniques.

Proposition d'un référentiel issu des analyses (Tourmen, 2007, p. 319-338)

Encadré 1 : Méthodologies utilisées dans les recherches concernées

\subsubsection{Définir et caractériser les situations génériques}

\section{de l'activité professionnelle}

Pour analyser les traces ainsi recueillies puis repérer et caractériser les situations génériques des activités professionnelles ciblées, il faut répondre à la question suivante : de quoi sont faites les situations?

On peut caractériser les situations de deux manières : par le donné des situations ou par leur niveau de complexité. 
Une situation de travail, ce sont d'abord des buts, emboîtés et souvent de natures différentes. Ces buts sont indissociables des objets matériels ou immatériels de l'action : ce sur quoi il y a à agir. L'action se réalise dans des conditions qui influencent le " comportement " des objets de l'action et les modalités de sa réalisation : conditions matérielles, techniques, naturelles, sociales, c'est-à-dire, ce qui doit être pris en compte et ce sur quoi il y a à agir pour réguler les actions.

Un certain nombre d'éléments se combinent pour rendre une situation plus ou moins complexe : nombre et types de buts à atteindre, situation à faible ou forte variabilité, résultats productifs immédiats ou prenant en compte les effets à moyen et long terme, conflit entre résultats productifs et protection de soi. À ces éléments s'ajoutent des variables essentielles à prendre en compte qui sont plus ou moins nombreuses, accessibles, prévisibles, pouvant constituer un système plus ou moins interactif.

Nous nous sommes appuyés sur ces caractéristiques pour identifier et décrire les situations génériques dans les domaines professionnels concernés. Nous les avons ainsi caractérisées par un intitulé, un ou des buts fixés, des objets à traiter et leurs caractéristiques essentielles pris en compte par les professionnels, des conditions de réalisation et enfin, des ressources allouées.

Sur la base des données recueillies dans les trois recherches, nous avons donc commencé par lister certaines des "situations génériques ", ou classes de situations, propres à l'activité professionnelle visée.

\section{Référentiel de situations du BTS aquaculture (Ferron, Mayen et al., 2006)}

Une liste de dix situations génériques a été établie par le groupe de travail dont voici quelques exemples d'intitulés : la gestion de l'oxygène (concentration) ; la gestion des équipements ; la gestion du cheptel (de l'espèce produite) ; le pilotage quotidien de l'entreprise (gestion).

\section{Référentiel de situations de l'ingénieur agronome (Métral \& Mayen, 2008)}

Dix situations génériques ont été identifiées et retenues par le groupe de travail : six ont pu faire l'objet d'une caractérisation, dont les trois données en exemple, ci-dessous :

\begin{tabular}{|l|l|}
\hline $\begin{array}{l}\text { Dénomination } \\
\text { de la situation générique } \\
\text { (ou classe de situations) }\end{array}$ & But de la situation générique \\
\hline Conception d'un projet & $\begin{array}{l}\text { Mettre en place les conditions nécessaires à la } \\
\text { réalisation d'un projet [...] }\end{array}$ \\
\hline $\begin{array}{l}\text { Conduite d'un projet (phase de } \\
\text { mise en œuvre du projet) }\end{array}$ & $\begin{array}{l}\text { Opérationnaliser, organiser et suivre la mise en } \\
\text { œuvre du plan d'action d'un projet. }\end{array}$ \\
\hline
\end{tabular}




\begin{tabular}{|l|l|}
\hline Encadrement d'une équipe & $\begin{array}{l}\text { Assurer, " au quotidien " et dans la durée, } \\
\text { l'encadrement d'une équipe de plusieurs personnes } \\
\text { sous sa responsabilité hiérarchique et la faire évoluer } \\
\text { dans son organisation [...] }\end{array}$ \\
\hline
\end{tabular}

3. Référentiel de situations des professionnels de l'évaluation de politiques publiques (Tourmen, 2007)

Huit situations génériques ont été identifiées parmi lesquelles : concevoir une commande d'évaluation dans une administration ; concevoir un projet d'évaluation en réponse à une commande ; piloter une équipe d'évaluateurs ; réaliser le recueil de données d'une évaluation ; communiquer les résultats d'une évaluation.

\section{Encadré 2. Exemples de situations génériques identifiées ${ }^{1}$}

Nous avons ensuite orienté nos analyses vers l'identification des "variables (ou dimensions) de situation agissantes " prises en compte par les sujets ayant une activité efficace (Pastré, Mayen \& Vergnaud, 2006). Ce sont celles que les professionnels prennent en compte pour diagnostiquer l'état d'une situation et qu'ils cherchent aussi à transformer. Ces variables sont présentes dans toutes les situations singulières mais prennent des valeurs ou états différents selon les cas (voir les exemples dans l'encadré 3). Nous avons aussi parfois été jusqu'à identifier des " indices" permettant de diagnostiquer l'état de ces variables en situation.

\section{Référentiel de situations du BTS aquaculture}

La situation générique " gérer l'oxygène " peut donner lieu à la prise en compte des variables suivantes : taux d'oxygène dans l'eau; type d'installation (bassins circulaires fonctionnant en circuit fermé, etc.) ; type d'eau (eau douce, eau de mer) ; espèces de poissons, etc.

\section{Référentiel de situations de l'ingénieur agronome}

Dans ce référentiel, la formalisation des variables agissantes de la situation " conception d'un projet " a pris la forme d'un schéma. II reprend les variables de la classe de situations relevant des acteurs (nombre d'acteurs, statut et poids, etc.), des objets (exigences de résultats, risques, etc.) et des conditions de l'action (moyens à disposition, etc.). Nous y avons également fait figurer les buts de la situation, ainsi que les rôles attribués au professionnel.

1 Nous présentons les résultats de nos travaux tels qu'ils apparaissent dans les documents selon les conventions en vigueur dans les institutions avec et pour lesquelles nous avons travaillé, ce qui explique le caractère parfois hétérogène des tableaux. 


\section{Référentiel de situations des professionnels de l'évaluation de politiques publiques}

Concernant la situation générique " concevoir une commande d'évaluation dans une administration ", nous avons identifié que les professionnels mobilisaient des concepts visant à assurer la " faisabilité " et la " légitimité " d'une évaluation. Pour les anticiper et les construire, les professionnels expérimentés prélèvent des indices et tentent d'agir sur les caractéristiques de trois variables de situation : l'objet à évaluer, les stratégies des acteurs autour de l'évaluation et les moyens pour évaluer. Chaque variable peut être caractérisée par des indices variés : la variable " moyens pour évaluer " se caractérise, par exemple, par l'ampleur du budget, du temps et des informations disponibles, la fiabilité des outils de recueil de données, la capacité des acteurs à coopérer ; la variable "stratégies des acteurs " se mesure par le statut des acteurs concernés, leur degré d'exigence méthodologique, l'urgence de leurs demandes, ses finalités, leurs possibles rejets de l'évaluation, etc.

Encadré 3. Exemples de variables de situation agissantes

\subsubsection{Décrire les actions habituellement réalisées par les professionnels}

Dans deux des référentiels, nous avons aussi identifié, pour chaque situation générique, un ensemble " d'actions réalisées " par les professionnels et quelques-uns des critères de réussite qui peuvent leur être adjoints. Ces actions sont à la fois gestuelles, mentales, langagières, et visent à diagnostiquer l'état d'une situation et à en transformer certaines dimensions.

Par exemple, dans le cas des évaluateurs de programmes et politiques publiques, la situation " concevoir une commande d'évaluation dans une administration " appelle la réalisation de tout ou partie des actions suivantes : diagnostiquer une situation d'évaluation (expliciter, clarifier et éventuellement reformuler la demande politique ou administrative, s'informer sur l'objet à évaluer et les moyens à disposition), rédiger un mandat/projet d'évaluation avec les acteurs impliqués, concevoir un dispositif d'évaluation, rédiger un cahier des charges d'évaluation, faire paraître un appel d'offres, examiner les propositions reçues et sélectionner des prestataires en charge de l'évaluation (en cas de prestataires externes), et au besoin, revoir la commande. Parmi les critères de réussite identifiés, on peut citer le fait que la démarche d'évaluation soit explicitée et négociée avec les acteurs concernés, ou encore, le fait qu'elle soit faisable, à savoir réalisable dans les contraintes de temps et de budget fixées.

Nous avons évité d'aller davantage dans le détail des actions à réaliser, pour limiter le risque de procéduraliser l'activité et de l'enfermer dans la reproduction d'une suite toujours identique d'actions et de faire de la formation une simple transmission de protocoles prescrits et de l'évaluation une simple vérification de conformité à des protocoles établis, négligeant par là même toute l'intelligence de l'adaptation aux situations. 


\subsubsection{Identifier les ressources mobilisées par les professionnels}

Enfin, nous avons tenté d'identifier des " ressources" (connaissances et concepts, modes de raisonnement, habiletés techniques et gestuelles) qui orientent et rendent possibles les actions (voir les exemples dans l'encadré 4). Elles permettent un certain niveau d'activité et s'y développent dans un même mouvement. Les " concepts pragmatiques " (Pastré, 1999) et autres connaissances faisant partie de ces ressources ont, ici, vocation à instrumenter la compréhension des situations et l'action dans et sur les situations.

\section{Référentiel de situations de l'ingénieur agronome}

Chez les ingénieurs, les ressources mobilisées apparaissent telles que les jurys de VAE les ont énoncées. Ceux-ci distinguent d'une part, les connaissances et outils conceptuels ou matériels, d'autre part, les " capacités " de l'ingénieur. Le référentiel donne aussi des exemples d'indicateurs utilisés par les jurys dans leur évaluation de ces ressources.

\begin{tabular}{|l|l|l|}
\hline Ressources & $\begin{array}{l}\text { Critères } \\
\text { (tels qu'utilisés dans les } \\
\text { jurys) }\end{array}$ & $\begin{array}{l}\text { Indicateurs } \\
\text { (non exhaustifs et à titre } \\
\text { d'exemple) }\end{array}$ \\
\hline $\begin{array}{l}\text { Connaître et intégrer à } \\
\text { ses raisonnements les } \\
\text { différentes dimensions } \\
\text { et enjeux d'un problème, } \\
\text { situation et/ou sur son } \\
\text { activité pendant l'action } \\
\text { d'un événement pour } \\
\text { prendre des décisions ou } \\
\text { proposer une solution à un } \\
\text { problème. }\end{array}$ & $\begin{array}{l}\text { Ne pas se contenter de } \\
\text { décrire ce que l'on a fait } \\
\text { et le résultat obtenu, } \\
\text { mais pouvoir expliquer } \\
\text { comment et en fonction } \\
\text { de quoi on a agi, quelles } \\
\text { dimensions on a prises } \\
\text { en compte (contraintes } \\
\text { économiques, technico- } \\
\text { économiques, humaines, } \\
\text { et pas seulement } \\
\text { techniques). }\end{array}$ \\
\hline $\begin{array}{l}\text { Mobiliser des } \\
\text { connaissances et outils } \\
\text { de l'ingénieur en } \\
\text { correspondance avec ses } \\
\text { fonctions et son domaine } \\
\text { d'activité [...] }\end{array}$ & $\begin{array}{l}\text { Maîtriser et mobiliser des } \\
\text { connaissances théoriques } \\
\text { pluridisciplinaires en } \\
\text { correspondance avec ses } \\
\text { fonctions [...] }\end{array}$ & $\begin{array}{l}\text { Utiliser des connaissances } \\
\text { et outils scientifiques } \\
\text { et techniques dans ses } \\
\text { réponses explications, } \\
\text { pour argumenter ses choix, } \\
\text { expliquer des phénomènes } \\
\text { ou processus observés, } \\
\text { critiquer ses conclusions } \\
\text { [...] }\end{array}$ \\
\hline
\end{tabular}




\section{Référentiel de situations des professionnels de l'évaluation de politiques publiques}

Parmi les ressources conceptuelles utilisées par les évaluateurs, on peut citer des concepts pragmatiques liés au champ conceptuel de la " faisabilité " et de la "légitimité " d'une évaluation, des " théories spontanées" ou " connaissances en acte " portant sur les situations d'évaluation, des " règles d'action " sur la manière de les évaluer et enfin des " buts " visés et redéfinis en action.

Pour les décrire dans un référentiel, nous avons classé ces ressources eu égard aux objets qu'elles concernent : ressources " techniques " liées à la maîtrise des outils de recueil de données en sciences sociales, ressources " méthodologiques " liées à la maîtrise des méthodologies d'évaluation, ressources " politiques " liées à la prise en compte des stratégies des acteurs autour de l'évaluation, enfin ressources " réflexives " qui constituent autant de leçons que les professionnels ont tirées des évaluations passées.

Exemples :

\begin{tabular}{|c|c|c|}
\hline Ressources & Critères & $\begin{array}{l}\text { Indicateurs } \\
\text { (exemples issus des discours } \\
\text { des professionnels) }\end{array}$ \\
\hline $\begin{array}{l}\text { Techniques : utiliser } \\
\text { des outils de recueil de } \\
\text { données en sciences } \\
\text { sociales }\end{array}$ & $\begin{array}{l}\text { Savoir réaliser des enquêtes } \\
\text { par questionnaires/ } \\
\text { entretiens/études de } \\
\text { cas, etc. } \\
\text { Connaître les propriétés } \\
\text { de faisabilité des } \\
\text { outils d'enquête (coût, } \\
\text { temps, etc.), leur pertinence } \\
\text { et efficacité selon les } \\
\text { publics. }\end{array}$ & $\begin{array}{l}\text { "on sait qu'avec certaines } \\
\text { cibles les entretiens } \\
\text { téléphoniques, ça ne } \\
\text { marche pas, il faut faire du } \\
\text { face-à-face". }\end{array}$ \\
\hline $\begin{array}{l}\text { Politiques : anticiper et } \\
\text { comprendre les stratégies } \\
\text { habituelles des acteurs } \\
\text { autour d'une évaluation }\end{array}$ & $\begin{array}{l}\text { Connaître les stratégies } \\
\text { habituelles ou possibles } \\
\text { d'utilisation des résultats, } \\
\text { mais aussi de pression/ } \\
\text { rejet/non acceptation, etc. }\end{array}$ & $\begin{array}{l}\text { "On a le pôle des opérateurs } \\
\text { qui veulent l'évaluation pour } \\
\text { pouvoir continuer à financer } \\
\text { d'autres choses". }\end{array}$ \\
\hline $\begin{array}{l}\text { Réflexives : tirer des } \\
\text { leçons sur les conditions } \\
\text { de réussite et d'échec des } \\
\text { évaluations }\end{array}$ & $\begin{array}{l}\text { Avoir tiré des leçons sur } \\
\text { des configurations plus } \\
\text { ou moins favorables, les } \\
\text { pratiques plus ou moins } \\
\text { adaptées et efficaces, } \\
\text { les différents critères de } \\
\text { réussite d'une évaluation et } \\
\text { leur combinaison. }\end{array}$ & $\begin{array}{l}\text { "Ce n'est pas parce } \\
\text { qu'une évaluation est } \\
\text { méthodologiquement bonne } \\
\text { qu'elle sera automatiquement } \\
\text { prise en compte". }\end{array}$ \\
\hline
\end{tabular}

Encadré 4. Exemples de ressources qui peuvent être mobilisées 
Nous avons évité d'aller trop loin dans la description des ressources, pour limiter le risque de les réifier et d'ôter toute liberté d'interprétation, d'évolution et d'ajustement par les utilisateurs des référentiels au regard des particularités de leur propre situation et expérience. Selon la théorie des instruments (Rabardel, 1995), cela rendrait plus difficile l'instrumentation de ces artefacts.

\section{Conclusion}

Ce type de référentiel peut avoir des implications sur l'ingénierie des parcours de professionnalisation. Les situations décrites peuvent servir de base à la conception des parcours de formation afin de s'assurer que les personnes vont pouvoir faire l'expérience (en formation, en stage) des situations génériques et de leurs variations. Elles peuvent aussi être utilisées pour la conception d'outils de suivi de stage, de livrets de parcours, etc.

Les situations peuvent aussi constituer le fil conducteur d'une formation, permettant l'articulation des différentes situations pédagogiques selon une progression définie, par exemple : en introduisant progressivement des dimensions supplémentaires aux situations. Les " ressources " peuvent alors être travaillées tout au long du parcours et à plusieurs reprises. Une même ressource pourra aussi être développée dans plusieurs situations et à travers plusieurs postures et points de vue disciplinaires.

Ce type de référentiel peut servir pour la conception d'outils pédagogiques utilisés en séance de formation : études de cas, mises en situation par des jeux de rôle, simulations. Les formateurs peuvent s'inspirer des éléments de situation listés pour construire leurs études de cas et en faire varier les principales dimensions agissantes: les variables de situations, les actions à réaliser et les ressources à mobiliser constituent autant d'éléments qui pourront nourrir leur activité de médiation. Ils peuvent faire focaliser l'attention sur une dimension de la situation non encore prise en compte, introduire, modifier ou retirer des ressources à la disposition des formés, ajouter des contraintes, changeant ainsi l'activité des sujets qui y sont engagés et ce qu'ils y construiront.

Enfin, un tel type de référentiel pourrait étayer la construction de situations évaluatives se référant aux situations de travail. Il contribuerait, par cette construction et par l'organisation des conditions de l'évaluation, à restituer le caractère vivant et dynamique des situations (diversité et variabilité, nature et niveau de complexité, etc.). Il viendrait appuyer l'activité évaluative des jurys, dans l'introduction de variations dans la situation, dans les questions adressées à l'évalué portant sur les variables essentielles de la situation ou encore dans les possibilités d'ouverture à d'autres modes de raisonnement et d'action pour que l'évaluation ne soit pas seulement l'espace d'application de manières d'agir stéréotypées. 
Nous souhaitons conclure sur une réserve. Quels que soient leur forme et leur contenu, les référentiels restent lacunaires en ce qui concerne le réel du travail et l'activité professionnelle visée. Ils ne peuvent épuiser l'ensemble des activités et des situations professionnelles d'un métier ou d'un emploi : "l'activité ne se laissera jamais enfermer dans un référentiel de compétences" (Prot, 2007). Un référentiel ne dispense pas ses utilisateurs de mobiliser leur propre connaissance " intime et en acte " du métier.

Patrick MAYEN

patrick.mayen@educagri.fr

Jean-François MÉTRAL jean-francois.metral@educagri.fr

Claire TOURMEN

claire.tourmen@educagri.fr

\section{BIBLIOGRAPHIE}

FERRON 0., HUMBLOT J.-P., MAYEN P. et al. (2006). "Introduire un référentiel de situations dans les référentiels de diplôme en BTS ", rapport de recherche de l'unité propre Développement professionnel et formation, département des sciences de la formation et de la communication, Établissement national d'enseignement supérieur agronomique de Dijon.

JONNAERT Ph., BARRETTE J., MASCIOTRA D. et al. (2006). La compétence comme organisateur des programmes de formation revisitée, ou la nécessité de passer de ce concept à celui de "l'agir compétent ", Montréal : actes du colloque de l'ORÉ.

LEPLAT J. (1991). "Compétences et ergonomie ", in R. Amalberti, M. de Montmollin \& J. Thereau (dir.), Modèles en analyse du travail, Liège : P. Mardaga, p. 263-278.

MAYEN P. (2004). " Le couple situation-activité, sa mise en œuvre dans l'analyse du travail en didactique professionnelle ", in J.-F. Marcel \& P. Rayou (dir.), Recherches contextualisées en éducation : biennale de l'éducation et de la formation, SaintFons : INRP.

MAYEN P. (2007). " Passer du principe d'alternance à l'usage de l'expérience en situation de travail comme moyen de formation et de professionnalisation ", Raisons Éducatives, $\mathrm{n}^{\circ}$ 1, p. 83-100.

MÉTRAL J.-F. \& MAYEN P. (2008). " De la VAE à la formation des ingénieurs : le référentiel de compétences situées comme instrument d'une approche par les compétences en formation ", rapport de recherche, Établissement national d'enseignement supérieur agronomique de Dijon.

PASTRÉ P. (1999). "La conceptualisation dans l'action : bilan et nouvelles perspectives ", Éducation permanente, $n^{\circ} 139$, p. 13-37. 
PASTRÉ P. (2002). "L'analyse du travail en didactique professionnelle ", Revue française de pédagogie, $\mathrm{n}^{0}$ 138, p. 9-17.

PASTRÉ P. (2004). "Introduction. Recherche en didactique professionnelle ", in R. Samurçay \& P. Pastré (dir.), Recherches en didactique professionnelle, Toulouse : Octarès, p. 1-13.

PASTRÉ P., MAYEN P. \& VERGNAUD G. (2006). "La didactique Professionnelle ", Revue française de pédagogie, $\mathrm{n}^{0}$ 154, p. 145-198.

PROT B. (2007). Communication au séminaire de recherche Vers une recomposition du métier d'enseignant, Unité Propre Développement professionnelle et Formation, Agrosup Dijon, 29-30 septembre 2008.

RABARDEL P. (1995). Les Hommes et les technologies : approche cognitive des instruments contemporains, Paris : A. Colin.

ROGALSKI J. (2004). "La didactique professionnelle : une alternative aux approches de " cognition située " et " cognitiviste " en psychologie des acquisitions", Activité, vol. 1, n 2, p. 103-120.

SAMURÇAY R. \& ROGALSKI J. (1993). Représentations de référence : outils pour le contrôle d'environnements dynamiques, in A. Weill-Fassina, P. Rabardel \& D. Dubois (dir.) Représentations pour l'action, Toulouse : Octarès éd., p. 183-208.

STROOBANTS M. (1994). "La visibilité des compétences ", in F. Ropé \& L. Tanguy (dir.), Savoirs et compétences: de l'usage de ces notions dans l'école et l'entreprise, Paris : I'Harmattan.

TOURMEN C. (2007). "Les compétences des évaluateurs. Le cas des évaluateurs de politiques publiques ", thèse de doctorat, université Grenoble 2-Pierre-Mendès-France.

TOURMEN C. (2008). "Les compétences des évaluateurs de politiques publiques ", Formation-Emploi, $\mathrm{n}^{0} 194$, p. 53-63.

VERGNAUD G. (1990). "La théorie des champs conceptuels", Recherches en didactique des mathématiques, $\mathrm{n}^{\circ} 10$ (2-3), p. 133-170.

VERGNAUD G. (1992). "Qu'est-ce que la didactique ? En quoi peut-elle intéresser la formation des adultes peu qualifiés ?", Éducation permanente, n ${ }^{0} 111$, p. 18-31.

VERGNAUD G. (1996). " Au fond de l'action, la conceptualisation ", in J.M. Barbier (dir.), Biennale de l'éducation et de la formation, Savoirs théoriques et savoirs d'action, p. 275-292, Paris: PUF.

VERGNAUD G. (2001). " Forme prédicative et forme opératoire de la connaissance ", Actes du Colloque GDM 2001, Jean Portugais (dir.), La notion de compétence en enseignement des mathématiques, analyse didactique des effets de son introduction sur les pratiques de la formation, Montréal. Disponible sur Internet : $<$ http://smf4.emath.fr/Enseignement/TribuneLibre/EnseignementPrimaire/ ConfMontrealmai2001.pdf>, consulté le 6 septembre 2010.

ZARIFIAN P. (2001). Le modèle de la compétence, Rueil-Malmaison : Éd. Liaisons. 


\section{Abstracts • Zusammenfassungen • Resúmenes}

\section{Work situations: references for systems of reference}

The aim of this paper is to propose an approach of reference systems starting from professional didactics and the founding place it gives work situations. In the field of professional training, situations come first because they are what professionals and future professionals have or will have to "sort out themselves". The need to identify and then characterize situations, particularly in the requirements they impose the activity, constitute a stage prior to the analysis and the description of the activity and even more prior to the analysis and the description of the resources which are useful to think and act in a real-life situation. In this approach work situations constitute the missing and little-known part of reference systems.

\section{Die Arbeitssituationen: Referenzen für die Bezugssysteme}

Dieser Artikel setzt sich als Ziel, einen Ansatz der Bezugssysteme anhand der Berufsdidaktik und der Gründungsrolle, die diese den Arbeitssituationen gewährt, vorzuschlagen. Bei der Berufsausbildung stehen die Situationen an erster Stelle, weil die Fachleute oder die zukünftigen Fachleute mit diesen „zurechtkommen” oder „zurechtkommen werden”. Die Notwendigkeit, die Situationen zu identifizieren und dann zu charakterisieren, besonders bei den für die Tätigkeit notwendigen Anforderungen, steht vor der Analyse und der Beschreibung der Tätigkeit und noch vor der Analyse und der Beschreibung der nützlichen Ressourcen um zu denken und handeln. In diesem Ansatz sind die Arbeitssituationen der abwesende und verkannte Teil der Bezugssysteme.

\section{Las situaciones de trabajo : referencias para los referenciales}

Este articulo tiene por meta proponer un acercamiento a los referenciales à partir de la didáctica profesional y del lugar fundador que ésta concede a las situaciones de trabajo. En formación profesional, lo primero son las situaciones porque son éstas en las cuales los profesionales y futuros profesionales, tienen o tendrán que " desenvolverse ". La necesidad de identificar y luego de caracterizar las situaciones, en particular en cuanto a las exigencias que imponen à la actividad, constituye una etapa anterior al análisis y à la descripción de la actividad, y aun más anterior al análisis y à la descripción de recursos útiles para pensar y actuar en situación. En este acercamiento, las situaciones de trabajo constituyen la parte ausente y mal conocida en los referenciales. 
